\title{
CÁLCULO DE DISTANCIA ENTRE LOS NODOS DE UNA RED INALÁMBRICA ZIGBEE EN FUNCIÓN DEL PARÁMETRO RSSI
}

Nahuel Vara, German A. Poletto, Dr. Manuel Cáceres, Dr. Arturo J. Busso $\left({ }^{*}\right)$

RESUMEN: Como respuesta al avance de la radiodifusión de bajo consumo, surge el protocolo Zigbee que permite la implementación de redes inalámbricas de microcontroladores, con topologías avanzadas y el acceso a parámetros involucrados en la comunicación, lo que permite la creación de las llamadas redes inteligentes.

De los parámetros de potencia involucrados en la comunicación se plantea la posibilidad de establecer la ubicación de los nodos de una red a partir de la triangulación, para lo cual es necesario inicialmente estimar la distancia entre ellos. Para este fin, se implementó una red Zigbee punto a punto con dos dispositivos Xbee Serie $2 \mathrm{PRO}$ con el objetivo de verificar la posibilidad de estimar la distancia entre ambos a partir del parámetro RSSI que es un indicador de la potencia percibida en la antena del dispositivo. Para ello se utilizan fórmulas empíricas ampliamente probadas en otras tecnologías inalámbricas como WiFi y Bluetooth.

De las muestras de RSSI obtenidas, se puede verificar que es posible estimar la distancia a partir de la potencia promedio con un error que disminuye a medida que los nodos se alejan, lo que permite obtener el orden de magnitud de la separación entre ambos.
Palabras claves: Zigbee, Xbee, RSSI, Microcontroladores, Red Inalámbrica de Sensores, WSN

INTRODUCCIÓN: Con el avance de la tecnología de comunicación empiezan a notarse las verdaderas ventajas de mantener los elementos de un sistema interconectados entre sí. El mundo de los microcontroladores no es ajeno a esto, y dicha tendencia tuvo como consecuencia el desarrollo de protocolos de comunicaciones y dispositivos pequeños, de bajo costo, inteligentes y lo suficientemente versátiles para adaptarse a las redes actuales.

En particular Zigbee es un protocolo de comunicación que ofrece la posibilidad de interconectar microcontroladores inalámbricamente y que además permite el acceso a parámetros muy importantes involucrados en la comunicación. Dicho protocolo es utilizado por los fabricantes de componentes electrónicos para la construcción de módulos de comunicación configurables y programables que permiten facilitar la implementación de una red de sensores (o WSN por sus siglas en ingles), entre otras aplicaciones.

Así, una red de sensores está compuesta por los llamados nodos, que son dispo-

$\left({ }^{*}\right)$ Grupo Energías Renovables - FaCENA - UNNE - Av. Libertad 5470, CP 3400, corrientes, Argentina tel. +54 (0)3783 473931 ext. 116 - e-mail: nahuel.vara@gmail.com 
sitivos generalmente pequeños, equipados con sensores, microcontroladores y radiotransmisores, por lo que un nodo no solo tiene la capacidad de medir un parámetro, sino también de procesar información y comunicarse con otros nodos.

Es importante en una red de sensores, conocer la ubicación de los mismos para darle significado a las variables medidas. Si bien en la actualidad existen módulos externos que permitirían la localización de un nodo con una buena precisión, estos resultan costosos y muchas veces el consumo extra que incorporan al nodo resulta en un problema.

Las características y la disponibilidad que ofrece Zigbee de la información que hace a la comunicación, como ser la potencia de recepción y transmisión, establece la posibilidad de estimar la distancia existente entre un nodo y otro, lo que a su vez permitiría incluir la función de localización física de los nodos de una red a partir del cálculo de triangulación.

Con el objetivo de establecer los estudios preliminares para lograr la localización de nodos en una red Zigbee a partir de la triangulación, se verificó la posibilidad de estimar la distancia entre dos nodos en función de la potencia de la señal realizando mediciones de potenciadistancia y definiendo el error cometido.

\section{EL PARÁMETRO RSSI}

Uno de los parámetros que el protocolo Zigbee dispone es el indicador de potencia de señal recibida o RSSI por sus siglas en inglés (Received Signal Strength Indicator) que es una escala de referencia utilizado para medir la potencia en la antena del módulo receptor asociado al último paquete de información recibido. Este parámetro está ampliamente estudiado ya que es también utilizado en tecnologías como WiFi y Bluetooth. En el caso particular de los módulos de comunicación Xbee (fabricados por Digi) que utilizan el protocolo Zigbee, la referencia utilizada es de $1 \mathrm{~mW}$ y la unidad es el $\mathrm{dBm}$.

De la teoría electromagnética es sabido que la potencia de la señal disminuye con el cuadrado de la distancia según lo indica la ecuación de Friis para transmisión en el espacio libre (C. A. Balanis (2005)). Sin embargo, existen ecuaciones para el parámetro RSSI basadas en datos empíricos (Oguejiofor (2013)) que establecen que la perdida de propagación de la señal recibida por un nodo a una distancia $d_{i}$ del nodo transmisor es:

$$
P_{L}\left(d_{i}\right)[d B]=P_{L}\left(d_{0}\right)[d B]+10 n \log _{10} \frac{d_{i}}{d_{0}}
$$

Donde $\mathrm{P}_{\mathrm{L}}\left(\mathrm{d}_{0}\right)$ es la perdida de propagación a una distancia de referencia conocida $d_{0}$ (generalmente $\left.d_{0}=1 \mathrm{~m}\right)$. $n$ es una constante que depende del medio y de los obstáculos que existan entre los nodos. En el espacio libre $n=2$, sin embargo, en un entorno real la propagación de la señal se ve afectada por fenómenos de reflexión, difracción y dispersión debido a los obstáculos del entorno, por lo que debe ser medida empíricamente.

De la ecuación anterior se tiene que:

$$
n=\frac{P_{L}\left(d_{i}\right)-P_{L}\left(d_{0}\right)}{10 \log _{10} \frac{d_{i}}{d_{0}}}
$$

Dicha ecuación permite la estimación de la constante $\mathrm{n}$ a partir de la medición de potencia en las antenas de los nodos y la distancia que los separa.

Finalmente, la potencia de señal recibida será (E. Lau (2008)):

$$
R S S I[\mathrm{dBm}]=-10 n \log _{10} d+A[\mathrm{dBm}]
$$


Donde $\mathrm{n}$ es la constante de perdida ya mencionada, $\mathrm{d}$ es la distancia entre el nodo transmisor y el receptor en metros y $\mathrm{A}$ es el valor de RSSI en la entena de un receptora a una distancia de 1 metro del transmisor.

Despejando se obtiene:

$$
d=10^{-\frac{R S S I-A}{10 n}}
$$

Xbee es un módulo de comunicación para interconectar microcontroladores a través del protocolo Zigbee, sin embargo la comunicación entre el modulo y el microcontrolador, o el modulo y el ordenador encargado de configurar el dispositivo se realiza a través de una comunicación por puerto serial. Existen aplicaciones de computadora que permiten configurar, programar y testear los dispositivos Xbee para su caracterización.

Para la estimación de la distancia que separa dos módulos a través del parámetro RSSI se utilizó el software XCTU 6.1 desarrollado por Digi para módulos Xbee que incluye una herramienta llamada "Range Test” que facilita la lectura del parámetro RSSI entre dos o más módulos de una red.

Se utilizaron dos módulos Xbee Serie 2 PRO con antena monopolo configurados para una comunicación punto a punto.

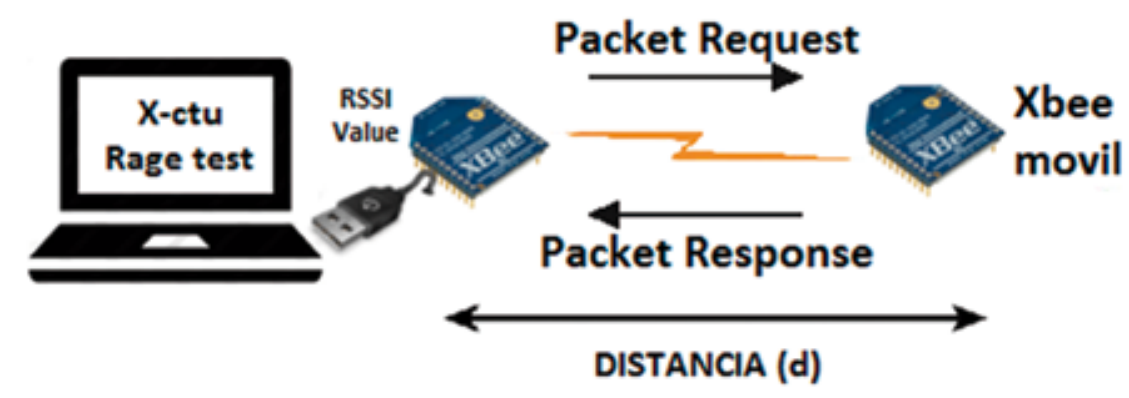

Figura 1: Diagrama de conexiones y enlaces utilizado/

El lugar donde se realizaron las mediciones fue la zona de canchas de futbol del Campus Universitario Deodoro Roca de la UNNE ubicado en la Avenida Libertad 5470 de la Ciudad de Corrientes. Dicho lugar permitió medir la potencia de las señales con los fenómenos de reflexión, difracción y dispersión disminuidos debido a la ausencia de obstáculos o elementos donde la señal pudiera reflejarse.

El proceso de medición consistió en fijar uno de los módulos en un sitio conveniente conectado a una computadora encargada de recabar los datos de potencia arrojados por el dispositivo y otro Xbee móvil conectado a una Tablet. Manteniendo fijas las orien- taciones relativas de los módulos para que la polaridad de las antenas fuera la misma en las distintas mediciones, se alejó uno de otro en línea recta. La distancia entre los módulos se midió con una cinta métrica.

La tarea del software utilizado consistió en el envío de un paquete de información por parte del módulo conectado a la computadora con destino al módulo ubicado a una distancia conocida, dicho paquete daba la orden a este último de informar al origen, es decir al Xbee conectado al ordenador, el valor de RSSI del último paquete recibido. El ordenador se encargaba de leer constantemente el valor de RSSI informado y de mostrarlo en pantalla en $\mathrm{dBm}$. Además 
realizaba un gráfico de RSSI en función del tiempo. Así, se registraron 8 valores de RSSI cada 5 metros de distancia entre los módulos a una tasa de 4 muestras por minuto y para una distancia de hasta 50 metros.

\section{RESULTADOS}

En primer lugar se midió el valor de RSSI a una distancia de 1 metro, necesario para utilizar la formula (2). Como puede verse en la siguiente tabla, $A=-36 \mathrm{dBm}$.

\begin{tabular}{|c|c|c|c|c|c|c|c|c|}
\hline $\begin{array}{c}\text { Distancia } \\
\text { (metros) }\end{array}$ & \multicolumn{70}{|c|}{ RSSI (dBm) } \\
\hline 1 & -36 & -36 & -36 & -36 & -36 & -36 & -36 & -36 \\
\hline
\end{tabular}

Tabla 1: Medición del parámetro A en dBm

De las mediciones de RSSI realizadas a distintas distancias, se obtuvieron los siguientes resultados:

\begin{tabular}{|c|c|c|c|c|c|c|c|c|c|c|}
\hline Distancia (metros) & 5 & 10 & 15 & 20 & 25 & 30 & 35 & 40 & 45 & 50 \\
\hline \multirow{5}{*}{ RSSI (dBm) } & -52 & -59 & -63 & -65 & -68 & -73 & -74 & -77 & -80 & -80 \\
\cline { 2 - 10 } & -51 & -60 & -61 & -64 & -69 & -71 & -78 & -79 & -77 & -82 \\
\cline { 2 - 10 } & -52 & -59 & -61 & -68 & -71 & -71 & -75 & -77 & -82 & -81 \\
\cline { 2 - 9 } & -53 & -59 & -65 & -68 & -70 & -73 & -72 & -77 & -80 & -80 \\
\cline { 2 - 9 } & -53 & -60 & -59 & -68 & -68 & -75 & -77 & -80 & -79 & -82 \\
\cline { 2 - 9 } & -53 & -58 & -61 & -65 & -69 & -76 & -75 & -79 & -80 & -83 \\
\hline
\end{tabular}

Tabla 2: Valores de RSSI medidos para distintas distancias de separación entre nodos

A partir de esta tabla es posible determinar la constante n dado por la ecuación (1). En promedio $\mathrm{n}=2,5682$.

Con las constantes calculadas es posible establecer finalmente los valores de distancias en función de los datos de RSSI con la formula (3). Se agrega además una fila correspondiente a las potencias promedio medidas que serán las usadas para calcular la distancia teórica y el error absoluto:

\begin{tabular}{|c|c|c|c|c|}
\hline $\begin{array}{c}\text { Distancia } \\
\text { Real }\end{array}$ & $\begin{array}{c}\text { RSSI Medido } \\
\text { Promedio }\end{array}$ & $\begin{array}{c}\text { Distancia } \\
\text { Teórica }\end{array}$ & $\begin{array}{c}\text { Error absoluto } \\
\text { (metros) }\end{array}$ & $\begin{array}{c}\text { Error } \\
\text { relativo }\end{array}$ \\
\hline 5 & $-52,333$ & 4,591 & 0,409 & 0,082 \\
\hline 10 & $-59,167$ & 7,981 & 2,019 & 0,202 \\
\hline 15 & $-61,667$ & 9,986 & 5,014 & 0,334 \\
\hline 20 & $-66,333$ & 15,174 & 4,826 & 0,241 \\
\hline 25 & $-69,167$ & 19,563 & 5,437 & 0,217 \\
\hline 30 & $-73,167$ & 28,002 & 1,998 & 0,067 \\
\hline 35 & $-75,167$ & 33,501 & 1,499 & 0,043 \\
\hline 40 & $-78,167$ & 43,841 & 3,841 & 0,096 \\
\hline 45 & $-79,667$ & 50,151 & 5,151 & 0,114 \\
\hline 50 & $-81,333$ & 58,234 & 8,234 & 0,165 \\
\hline
\end{tabular}

Tabla 3: RSSI medido promedio, distancia teórica y errores calculados a partir de las mediciones realizadas y las ecuaciones mencionadas. 
Es posible realizar una mejor comparación entre el valor real de la distancia de separación entre nodos y el teórico obtenido a partir de aplicar la fórmula (3) para los valores de RSSI medidos.

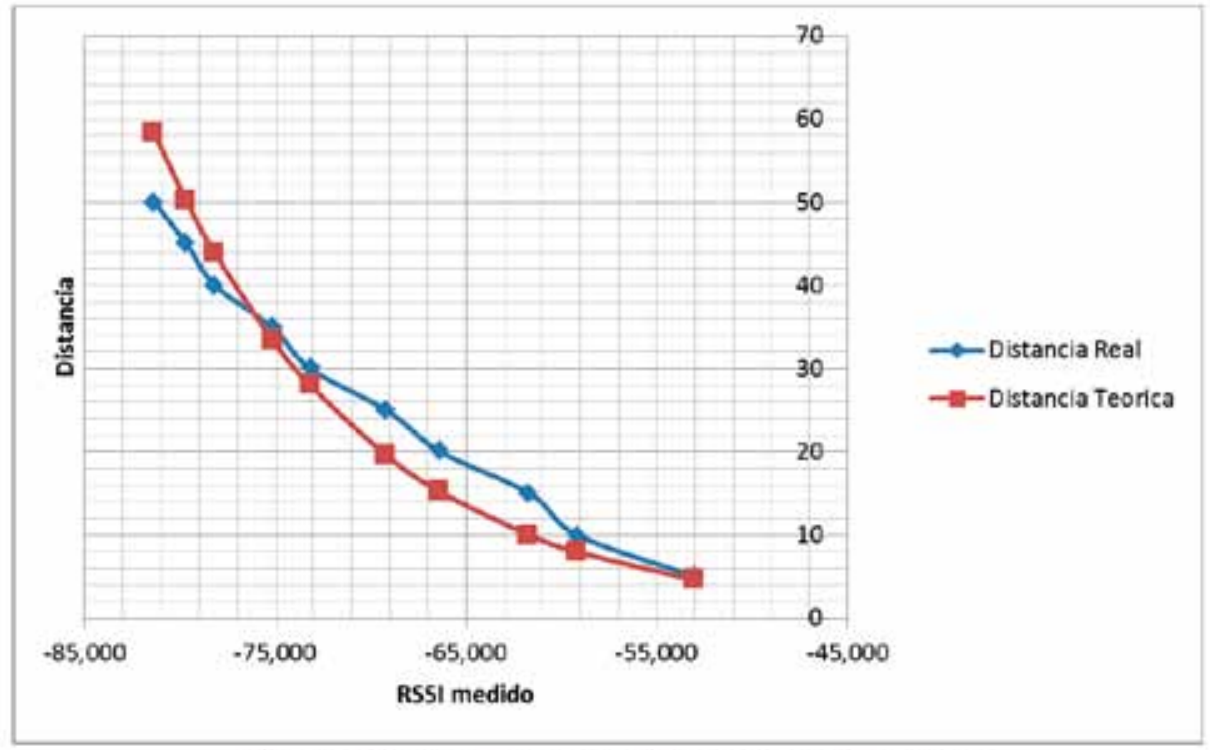

Figura 2: Distancia teórica y real en función del RSSI medido



Figura 3: Error relativo en función de la distancia. 


\section{CONCLUSIÓN}

Del análisis de los valores de distancia calculados a partir del muestreo del parámetro RSSI de un dispositivo Xbee Serie $2 \mathrm{PRO}$ se puede verificar que el mismo ha presentado errores de hasta 8 metros, sin embargo el error relativo cometido se ve claramente disminuido con la distancia, con lo cual, para distancias cortas (menores a 30 metros), el parámetro RSSI es útil para obtener el orden de magnitud de la distancia, y para separaciones mayores entre nodos, el valor de RSSI permite calcular la distancia con una precisión considerable.

Se debe aclarar que es el promedio de varias muestras del parámetro RSSI el que converge hacia un valor que permite un cál- culo de distancia estimado. Es decir, para lograr una estimación de la distancia, con los errores aquí expuestos, es necesario tomar varias muestras de potencia para una distancia fija entre nodos y utilizar el valor de RSSI promedio en la fórmula correspondiente.

Es importante considerar que el parámetro RSSI es totalmente accesible por cualquier nodo con un dispositivo Zigbee en funcionamiento, con lo cual las estimaciones de distancia entre ellos y su posterior localización por triangulación puede resultar en un nuevo recurso para una red en funcionamiento sin necesidad de incorporar nuevos dispositivos, lo que ampliaría las posibilidades de uso en determinadas aplicaciones.

\section{REFERENCIAS}

C. A. Balanis (2005). Antenna Theory, Analusis and Design. Wiley-Interscience. 3o Ed, CAP 2, pag 94.

E. Lau, B. Lee, S Lee, W. Chung (2008). Enhanced RSSI-Based high accuracy real-time user location tracking sistema for indoor and outdoor enviroments. International Journal On Smart Sensing And Intelligent System, VOL. 1, NO. 2, 534-548.

Oguejiofor O.S., Okorogu V.N., Adewale Abe, Osuesu B.O (2013). Outdoor Localization System Using RSSI Measurement of Wireless Sensor Network. International Journal of Innovative Technology and Exploring Engineering (IJITEE). ISSN: 2278-3075, Volume-2, Issue-2. 